\title{
A Sustainable Energy Investment Planning Model Based on the Micro-Grid Concept Using Recent Metaheuristic Optimization Algorithms
}

\author{
Soheil Mohseni \\ Sustainable Energy Systems, School of \\ Engineering and Computer Science \\ Victoria University of Wellington \\ Wellington 6140, New Zealand \\ https://orcid.org/0000-0001-7367-3757
}

\author{
Alan C. Brent \\ Sustainable Energy Systems, School of \\ Engineering and Computer Science \\ Victoria University of Wellington \\ Wellington 6140, New Zealand \\ https://orcid.org/0000-0003-3769-4512
}

\author{
Daniel Burmester \\ Sustainable Energy Systems, School of \\ Engineering and Computer Science \\ Victoria University of Wellington \\ Wellington 6140, New Zealand \\ https://orcid.org/0000-0003-4032-685X
}

\begin{abstract}
This paper develops an innovative sustainable energy investment planning framework to select the most economically viable option among a range of conceptual microgrid (MG) projects proposed to be implemented in an area, as well as the sizes of the associated components. It is assumed that the technical feasibility of a project is verified in advance based on the estimation of the potentials of renewable energy sources. Since the considered optimal capacity planning problem is not amenable to exact methods of optimization due to its nondeterministic polynomial-time hard (NP-hard) nature, metaheuristic optimization algorithms (MHOAs) are used in the devised optimum sizing approach. We demonstrate the applicability and efficacy of the proposed modelling framework based on three representative MGs in different topologies. The efficiencies of four newly introduced MHOAs viz. the moth-flame optimization algorithm, the sine-cosine algorithm, the multi-verse optimizer, and the water evaporation optimization algorithm are examined in solving the considered problem, whilst the hybrid genetic algorithm-particle swarm optimization is chosen as the benchmark algorithm. The simulation results indicate that the MFOA yields the highest quality solution sets in solving the MG design problems, and provides the most accurate estimate of the life-cycle costs of the MGs.
\end{abstract}

Keywords- Microgrids, Hybrid power systems, Power system planning, Heuristic algorithms, Particle swarm optimization

\section{INTRODUCTION}

Almost $20 \%$ of the world's population has no access to electricity [1]. Addressing this demand requires solutions that are both cost-effective and environmentally benign. Consequently, sustainable energy systems that use the potential of renewable energy sources (RES) are envisioned to provide reliable, affordable, and clean electricity to remote communities in an autonomous manner. If such systems are accurately designed, their long-term economic vitality could be ensured [2], [3].

Due to the non-linearity and non-convexity of the optimal design problem of the sustainable energy systems, and owing to the fact that the design variables could be of the type either discrete or continuous, a powerful optimization technique is required to solve such a complex problem. The multidimensional optimal design problems of sustainable energy systems, which are also subject to several linear and non-linear constraints, are not amenable to exact treatments if the design process is not simplified. In order to avoid jeopardizing the accuracy of the simulation results due to the model simplifications, the use of metaheuristic optimization algorithms (MHOAs) is strongly suggested in the literature. The successful application of several MHOAs to the problem at hand has been reported in many studies, among which, the following well-known algorithms can be mentioned: the particle swarm optimization (PSO) [4], the genetic algorithm (GA) [5], as well as the hybrid GA-PSO (HGAPSO) [6]. A detailed review of the optimization techniques applied to the optimum capacity planning of the renewable and sustainable energy systems can be found in [7]. However, because of the continuously developing nature of the MHOAs, and also the so-called no-free-lunch theorem [8], which postulates that there is no universally best MHOA covering a broad scope of problems, there is an on-going imperative to evaluate the performances of newly developed MHOAs in solving the optimum design problems of sustainable energy systems.

On the other hand, a major issue in the field of optimal design, sizing, and planning of the sustainable energy systems is that often the studies have determined the supremacy of an optimization algorithm, based only on the results obtained using a single renewable energy test system and simply generalized their findings across other systems. A crucial defect of such an inference regarding the succession of an optimization algorithm is that the results might not be the same, when the algorithms are applied to different sustainable energy system structures.

Based on the above premises, in this paper, we go beyond the state of the art by proposing a comparison framework to examine the performances of the MHOAs to be used in the optimal design studies of renewable and sustainable energy systems, which is sensitive to the topology of the test system. The main contributions of this study are as follows:

1. A generic sustainable energy investment planning modelling framework is proposed to select the best 
configuration of a micro-grid (MG), among all proposed cases to be implemented, for electrifying an area over a scheduled time horizon following an assessment on the renewable energy potential of the site under study.

2. A general MHOA-based optimal capacity planning approach is devised to calculate the optimum sizes of the components of MGs.

3. The performances of the four recently introduced MHOAs are compared with each other in terms of solution accuracy in the context of different MG structures.

The remainder of this paper is organized as follows. Section II briefly introduces the MHOAs, the performances of which for the optimal design and capacity planning of the sustainable energy systems are evaluated in this paper. Section III describes the MG configurations, used as test systems to examine the performances of the selected MHOAs. The proposed MHOA-based approach to optimally size the components of each MG structure is developed in Section IV. The case study application based on the three most fitting types of MGs for the considered site is demonstrated in Section V. Section VI concludes the paper. Finally, Section VII outlines the limitations of this study and suggests guidelines for future research.

\section{Selected Metaheuristics}

This section concisely reviews the MHOAs, whose efficacies in solving the optimal capacity planning problems of sustainable energy systems are under investigation. All the algorithms start the optimization procedure by creating a population as the search agents to be positioned randomly in the n-dimensional design space of the considered problem (in accordance with $\mathrm{n}$ design variables). An objective (fitness) function is then used to guide these agents towards the global optimum point through ranking their performances over the course of the search process. They also continue the optimization process until a maximum number of iterations as the stopping criterion is reached.

\section{A. Moth-Flame Optimization Algorithm}

The moth-flame optimization algorithm (MFOA) is a nature-based stochastic optimization technique, proposed by Mirjalili in 2015 [9]. The development of the algorithm was inspired by the so-called transverse orientation, which is the navigation system of moths at night. In this algorithm, both the moths and flames search the design space to find the global optima and the positions of the moths are updated with the guidance of the flames using the following logarithmic spiral function:

$$
S\left(M_{i}, F_{j}\right)=D_{i} e^{b r} \cos (2 \pi r)+F_{j},
$$

where $M_{i}$ represents the $i$-th moth; $F_{j}$ denotes the $j$-th flame; $D_{i}$ identifies the distance between the $i$-th moth and the $j$-th flame; $S$ indicates the spiral function; $b$ is a constant, which describes the shape of the logarithmic spiral, considered to be 1 in this paper; with $r$ being a random number within the range $[-1,1]$.

The algorithm uses the recent best solution sets obtained so far as the flames to lead the moths over the course of iterations and saves the fitness values of the best flame at each iteration as the best solution set approximated by the algorithm. This procedure is replicated $\mathrm{N}$ times, where $\mathrm{N}$ is the total number of function evaluations, which serves as the stopping criterion.

\section{B. Sine-Cosine Algorithm}

The sine-cosine algorithm (SCA), introduced by Mirjalili in 2016 [10], is a straightforward yet effective stochastic optimization technique based on the sine and cosine functions. The main strategy used in the SCA for updating the positions of the search agents can be mathematically expressed as (2).

$$
X_{i}^{t+1}= \begin{cases}X_{i}^{t}+r_{1} \times \sin \left(r_{2}\right) \times\left|r_{3} P_{i}^{t}-X_{i}^{t}\right|, & r_{4}<0.5 \\ X_{i}^{t}+r_{1} \times \cos \left(r_{2}\right) \times\left|r_{3} P_{i}^{t}-X_{i}^{t}\right|, & r_{4} \geq 0.5\end{cases}
$$

where $X_{i}^{t}$ represents the position of the $i$-th population member at the $t$-th iteration; $P_{i}^{t}$ is the position of the target point of the $i$-th search agent at the $t$-th iteration; $r_{1}$ is a random coefficient that dictates the movement direction of the elements and is obtained by (3); $r_{2}$ is a random factor within the range $[0,2 \pi]$ defining how far the movement should be towards or outwards the target point; $r_{3}$ is a random parameter to model the influence of the target point on the distance to be crossed; and $r_{4}$ is a random number in the range $[0,1]$.

$$
r_{1}=a-t \times \frac{a}{T}
$$

where $t$ denotes the current iteration, $T$ stands for the maximum number of iterations, and $a$ is a constant, the value of which is assumed to be 3 in this research study.

The best approximate solution sets of the considered problem over the course of iterations are recorded in a variable as the target point, the final value of which is returned at the end of the optimization process.

\section{Multi-Verse Optimizer}

The multi-verse optimizer (MVO) is another cutting-edge population-based stochastic MHOA, devised by Mirjalili et al. in 2016 [11]. The MVO takes inspiration from some of the cosmological theorems, including the big-bang theory, the theory of multiple universes, and the theory of general relativity. It also uses the notions of black holes, white holes, and wormholes to approximate the global optima through defining some rules. The mathematical formulation of the MVO algorithm is given by (4)-(6).

$$
\begin{aligned}
& x_{i}^{j} \\
& = \begin{cases}\left\{\begin{array}{l}
X_{j}+T D R \times\left(\left(u b_{j}-l b_{j}\right) \times r_{3}+l b_{j}\right), r_{2}<0.5 \\
X_{j}-T D R \times\left(\left(u b_{j}-l b_{j}\right) \times r_{3}+l b_{j}\right), r_{2} \geq 0.5
\end{array}\right. & r_{1}<W E P \\
x_{i, R W}^{j} & r_{1} \geq W E P\end{cases} \\
& T D R=1-\frac{l^{1 / p}}{L^{1 / p}}, \\
& W E P=c+l \times\left(\frac{d-c}{L}\right),
\end{aligned}
$$

where $x_{i}^{j}$ represents the position of the $i$-th candidate solution (universe) in the $j$-th dimension; $X_{j}$ denotes the best candidate 
solution found so far in the $j$-th dimension; $u b_{j}$ and $l b_{j}$ are the upper and lower bounds in the $j$-th dimension, respectively; $r_{1}$, $r_{2}$, and $r_{3}$ are random numbers within the range $[0,1] ; T D R$ stands for the travelling distance rate; WEP stands for the wormhole existence probability; $x_{i, R W}^{j}$ identifies the position of the $i$-th universe in the $j$-th dimension chosen using a roulette wheel selection strategy; $l$ is the current iteration; $L$ is the maximum number of iterations; $p$ is the exploitation accuracy (i.e. 6 in this paper); and $c$ and $d$ are constants, which in this paper equal 0.2 and 1 , respectively.

Finally, the best position of the candidate solution sets is reported, when the termination criterion is met.

\section{Water Evaporation Optimization}

The water evaporation optimization (WEO), put forward by Kaveh and Bakhshpoori in 2016 [12], is a novel swarmbased MHOA inspired by the evaporation of a miniscule amount of water molecules on solid surfaces. The algorithm mainly consists of the following three stages:

1) Monolayer Evaporation: In this stage, first, the fitness functions of the search agents (i.e. water molecules) are scaled to the interval $[-3.5,-0.5]$ by (7) and the substrate energy vectors are constructed.

$$
E_{\text {sub }}(i)^{t}=\frac{\left(E_{\max }-E_{\min }\right) \times\left(F i t_{i}^{t}-\operatorname{Min}(\text { Fit })\right)}{\operatorname{Max}(\text { Fit })-\operatorname{Min}(\text { Fit })}+E_{\text {min }}
$$

where $E_{\text {sub }}(i)^{t}$ represents the substrate energy vector of the $i$ th search agent at the $t$-th iteration; Fit $t_{i}^{t}$ is the fitness function of the $i$-th search agent at the $t$-th iteration; $E_{\max }$ (i.e. -0.5 ) and $E_{\min }$ (i.e. -3.5 ) are the maximum and minimum values of $E_{\text {sub }}$, respectively; and Max and Min are the maximum and minimum functions, respectively.

Then, the so-called monolayer evaporation matrix is constructed by (8).

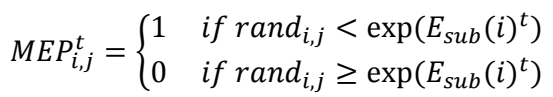

where $M E P_{i, j}^{t}$ represents the updating probability for the $j$-th variable of the $i$-th search agent at the $t$-th iteration and rand $_{i, j}$ is a number generated randomly from a uniform distribution between 0 and 1 .

2) Droplet Evaporation: In this stage, the fitness functions of the molecules are scaled to the interval $\left[-50^{\circ},-20^{\circ}\right]$ according to a procedure analogous to that described by (7). Then, the so-called droplet evaporation matrix $(D E P)$ is constructed.

3) Updating the Water Molecules: The next set of molecules can be generated as follows:

$$
W M^{(t+1)}=W M^{(t)}+S \times \begin{cases}M E P^{(t)}, & t \leq T / 2 \\ D E P^{(t)}, & t>T / 2\end{cases}
$$

where $S$ is a random permutation-based step size matrix, $t$ represents the current iteration, and $T$ denotes the maximum number of iterations. Then, for each water molecule, if the fitness function of the newly generated one is better than the previous one, the molecule's position will be updated to the new position. Subsequently, at each iteration, the best water molecule among all the molecules is selected. Finally, the algorithm terminates and the position of the best water molecule is returned if the stopping criterion is satisfied.

\section{E. Hybrid Genetic Algorithm-Particle Swarm Optimization}

The HGAPSO has been used as a benchmark optimization algorithm in this research study to evaluate the performances of the newly born MHOAs. The reason is that its efficiency in the context of the optimal planning of sustainable energy systems has been proven in [13] by comparing its performance with those of the most prevalent algorithms in this area, namely the basic GA and PSO.

For reasons of space, and also since the GA and PSO are well-known algorithms in this area, we omit their introduction. It must be noted that among different types of the HGAPSO, we use the algorithm that runs both of them in parallel. We refer the reader to [13] and the references therein for more details on the implementation of the HGAPSO technique.

Furthermore, the parameters of the HGAPSO are assigned as follows: the crossover and mutation probabilities in the GA part are chosen as 0.9 and 0.1 , respectively; while the learning factors and inertia weight in the PSO part are considered to be 2 and 0.7 , respectively. Moreover, a roulette wheel selection mechanism is adopted in the GA part of the hybrid algorithm to generate the parents.

\section{Micro-Grid Test Systems}

\section{A. Photovoltaic/Wind Turbine/Battery/Super-Capacitor}

The first MG test system, schematically diagrammed in Fig. 1, is a stand-alone $\mathrm{MG}$, which is equipped with photovoltaic (PV) panels and wind turbines (WTs) as power generation components, and incorporates a hybrid energy storage system, which consists of a battery bank and supercapacitors (SCs). The system also includes some power converters, among which the inverter that couples the residential loads to the MG's network is the only one whose size is going to be optimized independently. Note that the technical and financial specifications of the other converters shown inside the dashed lines in Fig. 1 are included in their respective components.

The mathematical modelling and description of the components of the MG test system 1 is illustrated below with instructions on the operation and power flow of the network.

The 175 W Shell Solar PowerMax Ultra (SQ175) PV panels, which feature monocrystalline PowerMax silicon solar cells are employed in this study. The power output from the PV generator at the $i$-th time slot can be calculated as in (10) [6].

$$
P_{P V}^{i}=N_{P V} \times \eta_{g} \times A_{m} \times G^{i},
$$

where $N_{P V}$ is the optimum number of PV panels approximated at each iteration of the optimization process; $\eta_{g}$ represents the overall efficiency of the PV generator, i.e. $13.3 \% ; A_{m}$ refers to the area of each PV panel, i.e. $1.31 \mathrm{~m}^{2}$; and $G^{i}$ denotes the solar irradiance $\left(\mathrm{W} / \mathrm{m}^{2}\right)$ at the $i$-th time slot. 
The WT generation system is the other power generator in this layout. The Northern Power NW100/19 WT is utilized in this study, which has a rated power of $100 \mathrm{~kW}$ AC. The power curve of each WT generator considered in this study is plotted in Fig. 2, which shows the relationship between its power output and the wind speed at the hub height (i.e. $25 \mathrm{~m}$ ) [14], [15]. The total amount of generated wind power at the $i$-th time slot can be calculated by multiplying the optimum number of WTs determined at each iteration of the optimization procedure by the wind power corresponding to the wind speed measured at that time slot, determined using the power curve of the WT.

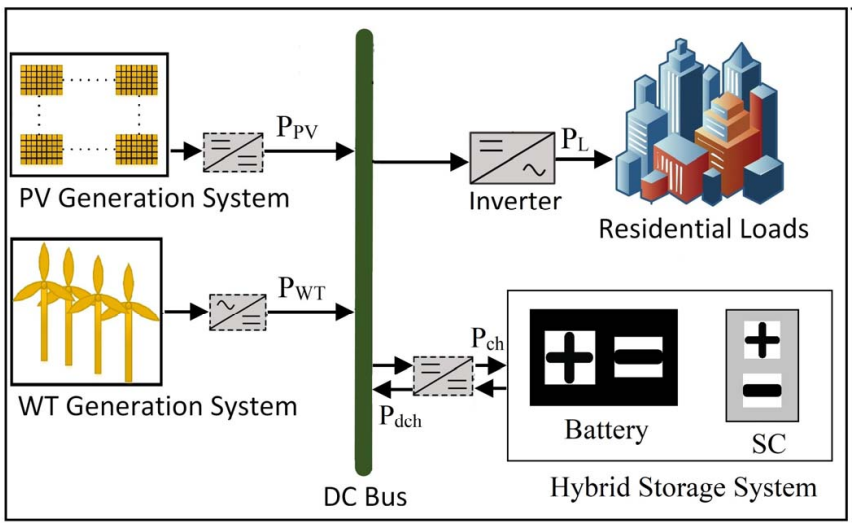

Fig. 1. Schematic diagram of the MG test system 1.

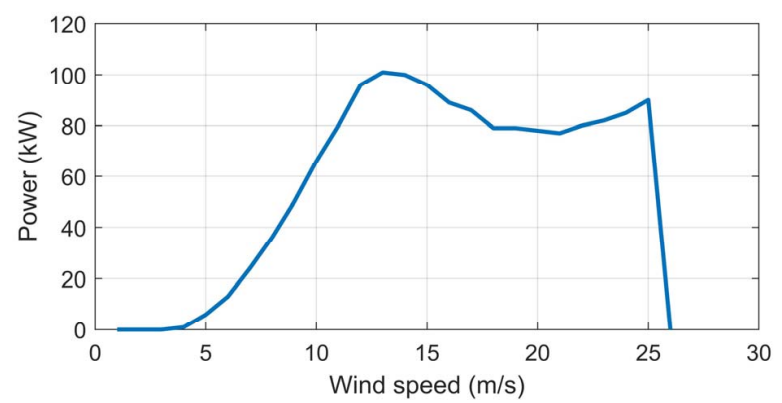

Fig. 2. Power curve of the Northern Power NW100/19 WT [15].

The energy storage system utilized in this configuration is a hybrid one to guarantee the dynamic stability of the system, which comprises of a battery bank and an SC bank offering mid-long- and short-term energy storage buffers, respectively, whose optimal capacities must be determined independently. The battery packs are considered to be of the advanced deep cycle lead acid battery type and of the product model Dryfit $\mathrm{A} 600 \mathrm{OPzV}$, whose rated capacity is $1.92 \mathrm{kWh}$ and is associated with an overall charging-discharging (round-trip) efficiency $\left(\eta_{b a t}\right)$ of $75 \%$ and its allowable depth of discharge is equal to 0.75 . The SC modules are considered to be generic ones with a nominal capacitance of $3500 \mathrm{~F}$ (i.e. equivalent to $\sim 3.23$ Wh considering a maximum current of $1500 \mathrm{~A}$ ) and a round-trip efficiency of $96 \%\left(\eta_{S C}\right)$. These components also impose inequality constraints on the operation of the system at each time slot, which integrate their minimum and maximum allowable capacities into the model. Note that the optimum number of battery packs and SC modules chosen at each iteration of the optimization protocol multiplied by their respective maximum allowable capacities represent the total capacities of the battery and SC banks, respectively.

In this paper, a low pass energy filter is used to determine the quotas of the battery and SC banks for backing up the renewable power generation, where the high-frequency component of the surplus/shortage of energy production must be stored/supplied by SCs, while the batteries take the responsibility to absorb/inject the low-frequency component of the energy excess/shortfall. Finally, the energy stored in the battery and SC banks at the $i$-th time slot can be expressed by the following equations:

$$
\begin{gathered}
E_{b a t}^{i}=E_{b a t}^{i-1}+\left(\left(P_{c h, L}^{i} \times \eta_{b a t}\right)-\left(P_{d c h, L}^{i} / \eta_{b a t}\right)\right) \times \Delta^{i}, \\
E_{S C}^{i}=E_{S C}^{i-1}+\left(\left(P_{c h, H}^{i} \times \eta_{S C}\right)-\left(P_{d c h, H}^{i} / \eta_{S C}\right)\right) \times \Delta^{i},
\end{gathered}
$$

where $P_{c h, L}^{i}$ and $P_{d c h, L}^{i}$ are the low-frequency components of the charging and discharging powers at the $i$-th time slot, respectively; $P_{c h, H}^{i}$ and $P_{d c h, H}^{i}$ are the high-frequency components of the charging and discharging powers at the $i$-th time slot, respectively; and $\Delta^{i}$ is the time slot increment $(1 \mathrm{~h})$.

Finally, the generic DC/AC inverter used to interface the residential loads with the MG network is modelled using its efficiency $\left(\eta_{i n v}\right)$, which is considered to be $95 \%$.

\section{B. Photovoltaic/Wind Turbine/Micro-Hydroelectric/Fuel Cell}

The second MG test system, depicted in Fig. 3, is an offgrid hydrogen-based MG system, which includes the PV, WT, micro-hydroelectric (MH), and fuel cell (FC) power generation units, and uses the hydrogen as the energy storage carrier. The hydrogen in this system is produced using an electrolyser, which can be stored in a hydrogen tank. Similar to the MG 1, the efficiencies and costs associated with all the power converters used within the structure of the system are included in their respective components, except for the residential loads' inverter. The mathematical models of the components of the system 2 are developed in the following paragraphs, together with the regulations on the operation of the system.

The PV panels, WTs, and the DC/AC inverter are modelled in a similar way as outlined for system 1.

A run-of-the-river hydropower scheme has also been considered in this system as a renewable power generation technology to supply the residential loads. The Natel FreeJet FJ-7A MH plant is used in this study to generate power, which has a rated power of $49 \mathrm{~kW}$, and the optimum number of which is going to be established. The power output from the MH plant $(\mathrm{kW})$ at the $i$-th time slot can be calculated as in (13) [16].

$$
P_{M H}^{i}=N_{M H} \times \rho \times h \times g \times \eta_{M H} \times \frac{F^{i}}{1000},
$$

where $N_{M H}$ represents the optimal number of $\mathrm{MH}$ plants computed at each iteration of the optimization protocol; $\rho$ is the water density, i.e. $1000 \mathrm{~kg} / \mathrm{m}^{3} ; h$ denotes the gross head height, i.e. $7 \mathrm{~m} ; g$ is the acceleration of gravity, i.e. $9.81 \mathrm{~m} / \mathrm{s}^{2}$; $\eta_{M H}$ is the efficiency of the $\mathrm{MH}$ system considering the AC/DC converter's efficiency, i.e. $55 \%$; and $F^{i}$ is the hourly streamflow at the $i$-th time slot $\left(\mathrm{m}^{3} / \mathrm{s}\right)$. 


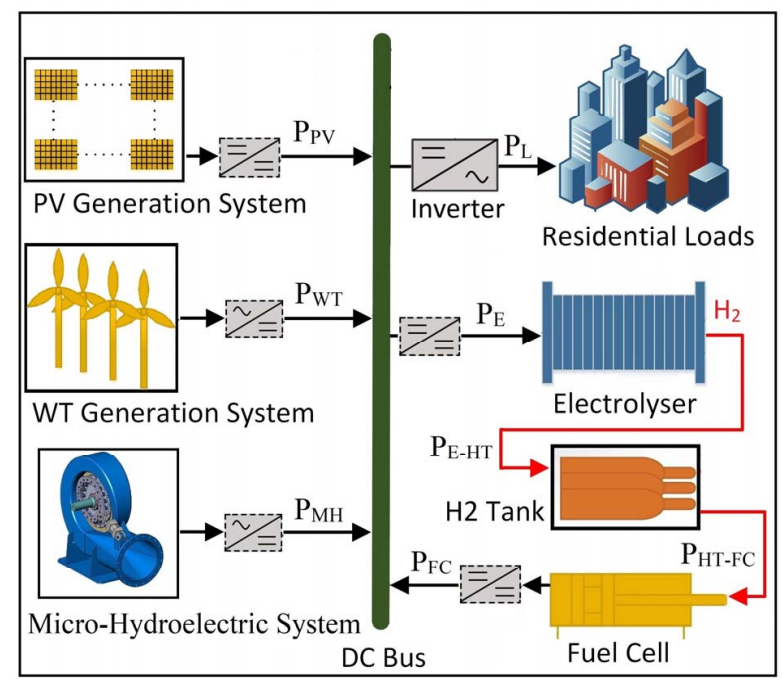

Fig. 3. Schematic diagram of the MG test system 2.

A hydrogen sub-system comprising of an electrolyser, a hydrogen tank, and an FC has also been embedded in this MG topology. The electrolyser draws the excess energy available from the RES to produce hydrogen, which is then stored in the hydrogen tank, while the FC has the role of providing the backup power to fulfil the residential demand when the power generated from the RES is not sufficient. In this study, the hydrogen sub-system is considered to be generic. The efficiencies of the electrolyser and FC taking into account the efficiencies of their respective converters are considered to be $60 \%$ and $40 \%$, respectively. Also, the round-trip efficiency of the hydrogen tank is assumed to be $95 \%$.

Furthermore, the hydrogen tank imposes some constraints on the operation and planning of the system: (1) the energy content of the tank at the end of the operational time span must be equal or more than its initial energy content, and (2) the energy content of the tank at each time slot of the operation must lie within a certain range to assure the admission of two following facts into the model: the inability of the tank to store the amounts of hydrogen beyond its allocated capacity (i.e. calculated at each iteration of the optimization process), and to release the amounts of hydrogen beyond its state of charge.

\section{Photovoltaic/Wind Turbine/Biomass/Flywheel}

The third MG test system, shown in Fig. 4, is also an isolated $\mathrm{MG}$, which utilizes the power generations from the PV, WT, and biomass generators, while incorporating a flywheel (FW) as an energy storage system. Likewise to the previous cases, the DC/AC inverter connecting the residential loads to the MG network is the only converter that participates in the optimal sizing procedure in an unsupervised fashion.

In the following paragraphs, we mathematically describe the performances of the components employed in the test system 3, along with some information on how to operate the system.

The mathematical models of the PV and WT generation systems, as well as the residential loads' inverter, are developed in a similar manner as outlined for system 1 .
The biomass power plant considered in this study consists of an anaerobic digestion reactor, a methane reformer, an air separation plant, and an internal combustion engine as an integrated set, the optimal power generation capacity of which is going to be calculated.

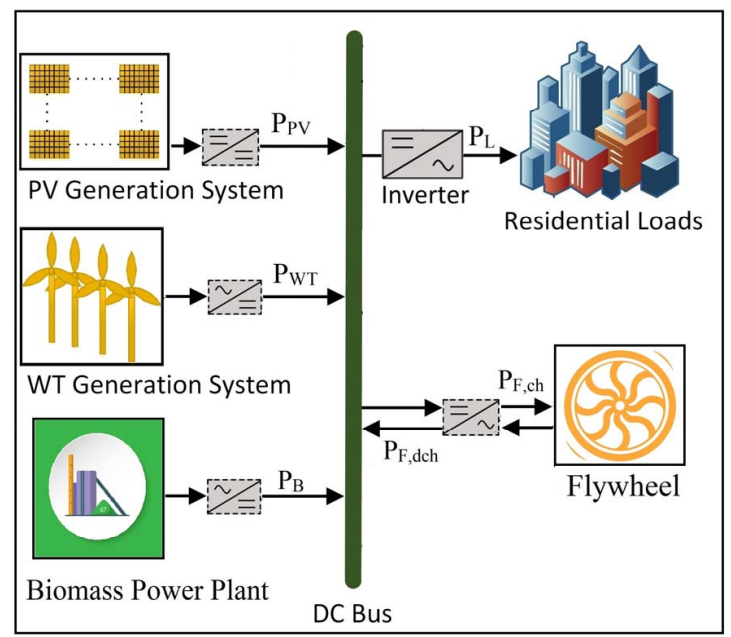

Fig. 4. Schematic diagram of the MG test system 3.

The anaerobic digestion reactor converts the wet biomass into the methane gas. The reformer then uses the methane to produce pure hydrogen gas based on the steam reforming process at high temperatures between 900 and $1000^{\circ} \mathrm{C}$. Finally, the hydrogen is burned with oxygen purified out of the air using the air separation plant in the internal combustion engine to generate clean electricity. Note that water is the only product of the combustion of hydrogen with oxygen. The overall waste $(\mathrm{kg})$ to power $(\mathrm{kW})$ efficiency of the plant, taking into account the efficiencies of the digestion reactor, methane reformer, and the hydrogen-fuelled internal combustion engine, is assumed to be $3.86 \%$, calculated by multiplying the efficiency of the reactor-reformer system proposed in [17], i.e. $4.54 \%$, by the efficiency of the hydrogen internal combustion engine proposed in [18], i.e. 85\%. Also, it is assumed that the wet waste is fed to the biomass power plant on a uniform basis, based on the available amount of waste on each day and, accordingly, the power generation profile of the biomass power plant is assumed to be uniform during the day. Furthermore, in this layout, the biomass plant does not engage in the optimum capacity planning practice, and its capacity is determined in advance based on the maximum daily amount of wet agricultural residues during the year in an independent way.

An FW is utilized in this system as an energy storage system, the optimum capacity of which must be determined. The FW stores the energy as kinetic in the form of a rotating rotor. The excess energy is stored within the FW through accelerating the rotor and rapidly increasing its speed, whereas the shortfall of energy is compensated for through deaccelerating the rotor and quickly decreasing its speed. Generally, the energy stored in an FW can be calculated as follows [19]:

$$
E_{F}=\frac{1}{2} I \omega^{2}
$$


where $I$ denotes the moment of inertia and $\omega$ represents the angular velocity of the rotor.

However, in our case, the amount of energy stored in the FW at the $i$-th time slot can be modelled as in (15).

$$
E_{F}^{i}=E_{F}^{i-1}+\left(\left(P_{F, c h}^{i} \times \eta_{F}\right)-\left(P_{F, d c h}^{i} / \eta_{F}\right)\right) \times \Delta^{i},
$$

where $P_{F, c h}^{i}$ and $P_{F, d c h}^{i}$ are the power flows injected/drawn to/from the FW, while $\eta_{F}$ denotes the round-trip efficiency of the FW, which is considered to be $80 \%$ for a generic FW. The FW also imposes two inequality constraints on the system in terms of its minimum and maximum allowable rotor speeds.

Moreover, it must be noted that in all the MG test systems above, a resistive dump load with a negligible total cost impact absorbs the surplus power beyond the capacities of the battery packs/SC modules/FW/electrolyser/hydrogen tank to keep the power balance in the MG networks.

\section{MHOA-BASEd Optimal Sizing Methodology}

In this paper, an MHOA-based sizing strategy is adopted to minimize the 20 year-round total costs of the MGs subject to the associated technical and operational constraints discussed in Section III. In this manner, we use the net present cost (NPC) concept, which calculates the present value of all the costs that each MG brings on over its expected lifetime (here, the capital, replacement, and operation and maintenance (O\&M) costs of the components), minus the present value of the total revenue that it collects over the project horizon (here, the salvage values of the components), utilizing the real interest rate notion. In this study, we have used the real interest rate of $5 \%$ to discount the future cash flows. The year 2019 is considered as the base year and the lifetimes of all the aforesaid projects are 20 years. For each test system, the summation of the NPCs of all the components, whose optimum sizes are under question, called the total NPC (TNPC), is selected as the objective function.

We have also used the equivalent loss factor (ELF) reliability indicator, which is modelled as a constraint element in the planning level, against which the optimization is undertaken. In this research study, the ELF is constrained to be lower than 0.01, as suggested in [17] for stand-alone MGs, which is enough for ensuring a reliable power supply to the considered case study. For considerations of space, we refer the reader to [17] and references therein for further discussion on the NPC and ELF methods.

Accordingly, for each MG, the optimum capacity planning method developed in this paper minimizes the TNPC against the predetermined reliability index (i.e. calculated by operating the MG using the operational strategy presented in Section III over the timeframe considered at each optimization iteration), using each of the MHOAs, whose performances are under investigation utilizing an average ranking index, through which the optimum sizes of the respective components of the MGs are calculated. The operational timeframe is initially considered to be one representative year in 1-hour intervals. Then, the year-round operational model is reduced to a $12 \times 24$ model, which uses the monthly averaged 24-h profiles for the meteorological and load demand input data to ease the computational effort.

\section{CASE STUdy}

\section{A. Input Data}

In order to evaluate the performance of the proposed approach, a case study analysis is carried out. A remoted community in the Feilding area, New Zealand (latitude $40.2253^{\circ} \mathrm{N}$, longitude $175.5675^{\circ} \mathrm{E}$ ), was selected as the case study site. The load profile is predicted for a population of 350 , according to the New Zealand GREEN grid household electricity demand study [20], which has a peak load demand of $695 \mathrm{~kW}$ occurring in July due to the maximum application of electric space heaters.

The heatmap like plots of the forecasted total residential load on the MG networks, the forecasted solar irradiance, and the forecasted wind speed at the considered case study site, in the form of monthly mean 24-h profiles, are shown in Figs. 57, respectively. The monthly mean profiles for the streamflow of the Rangitikei River that flows through the community's valley, as well as of the wet biomass assumed to be available at the site in metric tonnes per day, are shown in Fig. 8. Notice that the climatic data are forecasted based on the actual measured data at the Feilding area for a period of 15 years between 2003 and 2017, which were acquired from New Zealand's National Climate Database [21].

Also, notice that the wind speed data were monitored at 48 $\mathrm{m}$ above the ground level and scaled to the height of $25 \mathrm{~m}$ (in compliance with the Northern Power NW100/19 WT's hub height) according to the technique suggested in [14].

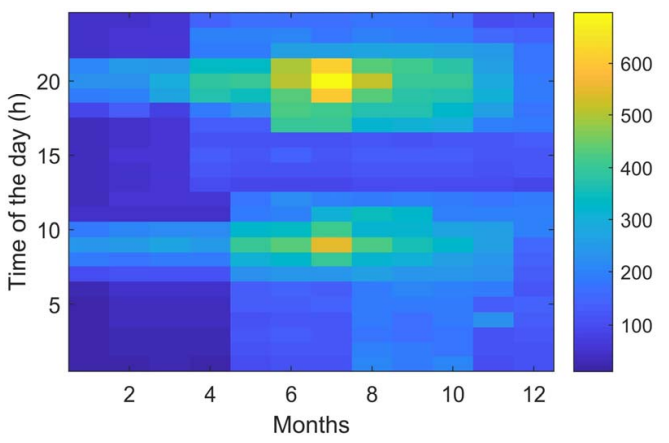

Fig. 5. Monthly mean 24-h profile for the residential loads (kW).

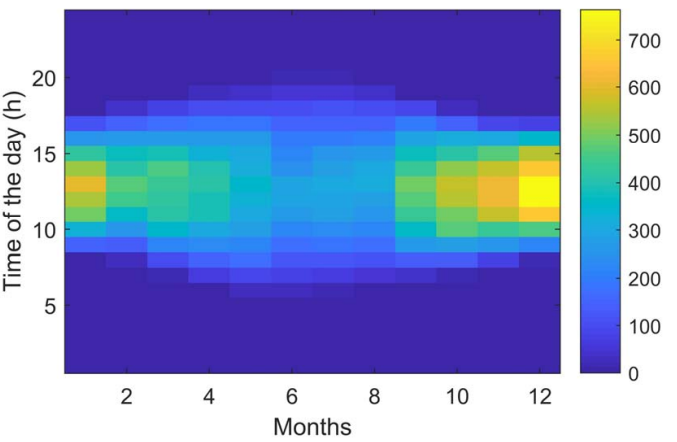

Fig. 6. Monthly mean $24-\mathrm{h}$ profile for the solar irradiance $\left(\mathrm{W} / \mathrm{m}^{2}\right)$.

Furthermore, Table I lists the data on the technical and financial specifications of the components utilized in the $\mathrm{MG}$ samples described above [13]-[18], [22], [23]. 


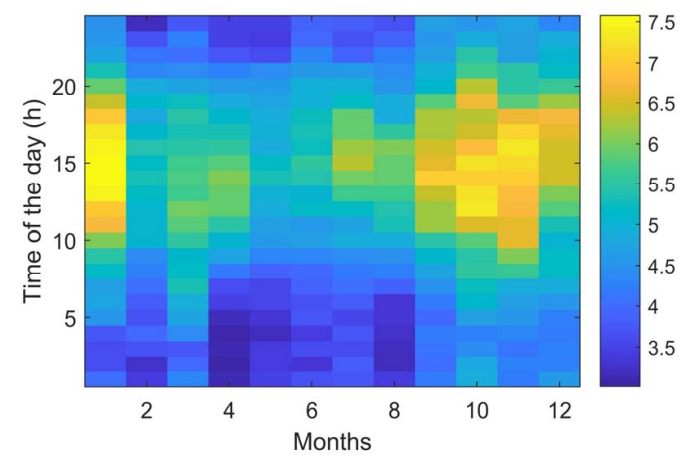

Fig. 7. Monthly mean 24-h profile for the wind speed $(\mathrm{m} / \mathrm{s})$.

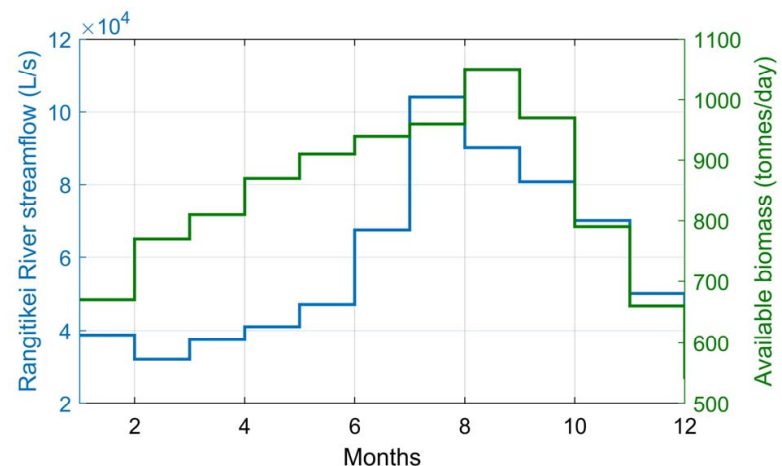

Fig. 8. Monthly mean profiles for the streamflow and available wet biomass.

TABLE I. SPECIFICATIONS OF THE COMPONENTS [13]-[18], [22], [23]

\begin{tabular}{cccccc}
\hline Component & $\begin{array}{c}\text { Product } \\
\text { model }\end{array}$ & $\begin{array}{c}\text { Capital } \\
\text { cost }\end{array}$ & $\begin{array}{c}\text { Replace- } \\
\text { ment cost }\end{array}$ & $\begin{array}{c}\text { O\&M } \\
\text { cost }\end{array}$ & $\begin{array}{c}\text { Life- } \\
\text { time } \\
\text { (year) }\end{array}$ \\
\hline PV panel & SQ175 & $\begin{array}{c}\$ 375 \\
/ \text { unit }\end{array}$ & $\begin{array}{c}\$ 375 \\
/ \text { unit }\end{array}$ & $\begin{array}{c}\$ 7.5 / \\
\text { unit-yr }\end{array}$ & 20 \\
\hline WT & NW100/ & $\begin{array}{c}\$ 110000 \\
/ \mathrm{unit}\end{array}$ & $\begin{array}{c}\$ 70000 \\
/ \mathrm{unit}\end{array}$ & $\begin{array}{c}\$ 2200 / \\
\text { unit-yr }\end{array}$ & 20 \\
\hline MH unit & FJ-7A & $\begin{array}{c}\$ 55000 \\
/ \mathrm{unit}\end{array}$ & $\begin{array}{c}\$ 55000 \\
/ \mathrm{unit}\end{array}$ & $\begin{array}{c}\$ 1100 / \\
\text { unit-yr }\end{array}$ & 25 \\
\hline Biopower & Generic & $\begin{array}{c}\$ 2150 \\
/ \mathrm{kW}\end{array}$ & $\begin{array}{c}\$ 2150 \\
/ \mathrm{kW}\end{array}$ & $\begin{array}{c}\$ 43 / \\
\mathrm{kW}-\mathrm{yr}\end{array}$ & 20 \\
\hline Battery & A600- & $\begin{array}{c}\$ 827 \\
/ \mathrm{unit}\end{array}$ & $\begin{array}{c}\$ 827 \\
/ \mathrm{unit}\end{array}$ & $\begin{array}{c}\$ 17 / \\
\text { unit-yr }\end{array}$ & 5 \\
\hline OPC & Generic & $\$ 62 / \mathrm{unit}$ & $\$ 62 / \mathrm{unit}$ & $\begin{array}{c}\$ 1.24 / \\
\text { unit-yr }\end{array}$ & 15 \\
\hline Flywheel & Generic & $\begin{array}{c}\$ 800 \\
/ \mathrm{kWh}\end{array}$ & $\begin{array}{c}\$ 800 \\
/ \mathrm{kWh}\end{array}$ & $\begin{array}{c}\$ 16 / \\
\mathrm{kWh}-\mathrm{yr}\end{array}$ & 15 \\
\hline Electrolyser & Generic & $\begin{array}{c}\$ 1000 \\
/ \mathrm{kW}\end{array}$ & $\begin{array}{l}\$ 1000 \\
/ \mathrm{kW}\end{array}$ & $\begin{array}{c}\$ 20 / \\
\mathrm{kW}-\mathrm{yr}\end{array}$ & 15 \\
\hline H2 tank & Generic & $\begin{array}{c}\$ 470 \\
/ \mathrm{kg}\end{array}$ & $\begin{array}{l}\$ 470 \\
/ \mathrm{kg}\end{array}$ & $\begin{array}{c}\$ 9.4 / \\
\mathrm{kg}-\mathrm{yr}\end{array}$ & 20 \\
\hline Fuel cell & Generic & $\begin{array}{c}\$ 2400 \\
/ \mathrm{kW}\end{array}$ & $\begin{array}{c}\$ 2400 \\
/ \mathrm{kW}\end{array}$ & $\begin{array}{c}\$ 48 / \\
\mathrm{kW}-\mathrm{yr}\end{array}$ & 5 \\
\hline Generic & $\begin{array}{c}\$ 750 \\
/ \mathrm{kW}\end{array}$ & $\begin{array}{c}\$ 750 \\
/ \mathrm{kW}\end{array}$ & $\begin{array}{c}\$ 15 / \\
\mathrm{kW}-\mathrm{yr}\end{array}$ & 15 \\
\hline
\end{tabular}

\section{B. Design Results}

In this paper, the MATLAB software is utilized to code and execute the proposed MHOA-based optimal sizing technique. Table II compares the performances of the MHOAs embedded in the framework of the proposed approach in terms of approximating the global optimum solution set based on the average results obtained over 30 independent runs. In the table, the acronym MTNPC stands for mean total net present cost. In this study, the comparisons are made only with regard to the mean solution accuracy and no attention has been paid to the computational expensiveness of the algorithms. This is because the considered sustainable MGs are going to be installed for a projected life span of 20 years in the studied site. This implies that a long computational time (in the order of several hours or even days) is tolerable, unless they rise computational intractability, which has not been the case in the metaheuristic algorithms analyzed in this study. Also, the maximum number of iterations, and the number of candidate solution sets for all the evaluated algorithms, are considered to be the same and equal to 350 and 60 , respectively.

TABLE II. Statistical Performance Comparison of the MHOAs

\begin{tabular}{ccccccc}
\hline \multirow{2}{*}{$\begin{array}{c}\text { Test } \\
\text { system }\end{array}$} & $\begin{array}{c}\text { MTNPC/ } \\
\text { Score }\end{array}$ & MFOA & SCA & MVO & WEO & $\begin{array}{c}\text { HGA- } \\
\text { PSO }\end{array}$ \\
\cline { 3 - 7 } MG 1 & $\begin{array}{c}\text { MTNPC } \\
(\$ M)\end{array}$ & 4.31 & 4.37 & 4.87 & 4.62 & 4.40 \\
\cline { 2 - 7 } & Score & 1 & 2 & 5 & 4 & 3 \\
\hline \multirow{2}{*}{ MG 2 } & $\begin{array}{c}\text { MTNPC } \\
(\$ M)\end{array}$ & 5.78 & 5.99 & 6.07 & 6.25 & 5.91 \\
\cline { 2 - 7 } & Score & 1 & 3 & 4 & 5 & 2 \\
\hline \multirow{2}{*}{ MG 3 } & $\begin{array}{c}\text { MTNPC } \\
(\$ M)\end{array}$ & 5.13 & 5.37 & 5.26 & 5.59 & 5.04 \\
\cline { 2 - 7 } & Score & 2 & 4 & 3 & 5 & 1 \\
\hline Average score & 1.33 & 3 & 4 & 4.66 & 2 \\
Final rank & 1 & 3 & 4 & 5 & 2 \\
\hline
\end{tabular}

Table II is interesting in several ways. Firstly, it shows that the first test system is by far a better option than the other two for the considered case study. This can be attributed to the technology matureness level of the components used within its structure (especially those integrated to supply backup power, namely the battery and SC banks), whose costs have reduced much in recent years. Secondly, it highlights the potential significance of the MFOA in minimizing the TNPCs of the MGs. Thirdly, it verifies our hypothesis concerning the inaccuracy of the generalization of the findings regarding the superiority of an MHOA according to the assessments undertaken for a single test system. According to the average scores given to each MHOA, we can rank the performances of the analyzed algorithms within the context of the study area as follows: the MFOA $>$ the HGAPSO > the SCA > the MVO > the WEO. In order to provide a better understanding of how the TNPCs are distributed among components, or in other words, the sizes determined by the considered algorithms, the optimum sizing results obtained for the three MGs are reported in Tables III-V, which represent the best-case performances of the adopted algorithms over 30 trials.

TABLE III. Best Performance Comparison of the MHOAs for the MG 1

\begin{tabular}{ccccccc}
\hline Algorithm & $\begin{array}{c}\text { PV } \\
\text { (no.) }\end{array}$ & $\begin{array}{c}\text { WT } \\
\text { (no.) }\end{array}$ & $\begin{array}{c}\text { Bat. } \\
\text { (no.) }\end{array}$ & $\begin{array}{c}\text { SC } \\
(\text { no. })\end{array}$ & $\begin{array}{c}\text { Inv. } \\
(\mathrm{kW})\end{array}$ & $\begin{array}{c}\text { TNPC } \\
(\$ \mathrm{SM})\end{array}$ \\
\hline MFOA & 941 & 5 & 141 & 17 & 561 & 4.18 \\
\hline SCA & 955 & 5 & 146 & 18 & 574 & 4.20 \\
\hline MVO & 1048 & 5 & 166 & 19 & 621 & 4.51 \\
\hline WEO & 987 & 5 & 159 & 18 & 595 & 4.36 \\
\hline HGAPSO & 966 & 5 & 152 & 17 & 585 & 4.23
\end{tabular}


TABLE IV. Best Performance Comparison of the MHOAs for the MG 2

\begin{tabular}{ccccccccc}
\hline Alg. & $\begin{array}{c}\text { PV } \\
(\text { no. })\end{array}$ & $\begin{array}{c}\text { WT } \\
(\text { no. })\end{array}$ & $\begin{array}{c}\text { MH } \\
(\text { no. })\end{array}$ & $\begin{array}{c}\text { Elec. } \\
(\mathrm{kW})\end{array}$ & $\begin{array}{c}\text { Tank } \\
(\mathrm{kg})\end{array}$ & $\begin{array}{c}\text { FC } \\
(\mathrm{kW})\end{array}$ & $\begin{array}{c}\text { Inv. } \\
(\mathrm{kW})\end{array}$ & $\begin{array}{c}\text { TNPC } \\
(\$ \mathrm{SM})\end{array}$ \\
\hline MFOA & 682 & 4 & 5 & 1119 & 902 & 277 & 566 & 5.61 \\
\hline SCA & 697 & 4 & 5 & 1195 & 908 & 285 & 588 & 5.70 \\
\hline MVO & 703 & 4 & 5 & 1204 & 915 & 291 & 590 & 5.73 \\
\hline WEO & 714 & 4 & 5 & 1239 & 932 & 310 & 601 & 5.86 \\
\hline $\begin{array}{c}\text { HGA- } \\
\text { PSO }\end{array}$ & 693 & 4 & 5 & 1174 & 912 & 284 & 579 & 5.67 \\
\hline
\end{tabular}

TABLE V. Best Performance Comparison of the MHOAs for the MG 3

\begin{tabular}{ccccccc}
\hline Algorithm & $\begin{array}{c}\text { PV } \\
\text { (no.) }\end{array}$ & $\begin{array}{c}\text { WT } \\
(\text { no. })\end{array}$ & $\begin{array}{c}\text { Bio. } \\
(\mathrm{kW})\end{array}$ & $\begin{array}{c}\text { FW } \\
(\mathrm{KWh})\end{array}$ & $\begin{array}{c}\text { Inv. } \\
(\mathrm{kW})\end{array}$ & $\begin{array}{c}\text { TNPC } \\
(\$ \mathrm{M})\end{array}$ \\
\hline MFOA & 748 & 5 & 170 & 309 & 568 & 4.89 \\
\hline SCA & 760 & 5 & 170 & 308 & 574 & 5.07 \\
\hline MVO & 755 & 5 & 170 & 314 & 570 & 4.96 \\
\hline WEO & 772 & 5 & 170 & 324 & 599 & 5.31 \\
\hline HGAPSO & 739 & 5 & 170 & 307 & 565 & 4.84 \\
\hline
\end{tabular}

\section{CONCLUSIONS}

This paper has introduced a mean-based adaptive scheme for quantifying the performances of MHOAs applied to infrastructure planning of MGs. In this light, the performances of the four recently developed MHOAs viz. the MFOA, the SCA, the MVO, and the WEO are assessed by applying them to three MG projects considered for a remote community in the Feilding area, New Zealand, while taking into account the HGAPSO as the standard algorithm. As the primary finding, we have acknowledged the possibility of arising dissimilar evaluations when MHOAs are applied to different case studies/MG systems, and accordingly, proposed a mean-based framework to assess the qualification of the algorithms. Furthermore, utilizing a statistical approach, we have revealed the proper performance of the MFOA in the optimal design of sustainable energy systems. Moreover, from the case study standpoint, a PV/WT MG equipped with a hybrid battery-SC energy storage system is shown to be the most cost-effective plan to electrify an additional population of 350 to be resettled in the Feilding area, New Zealand, whilst also addressing the dynamic stability concerns due to the presence of the high power density SC modules within its network.

\section{Limitations AND FutuRE ReSEARCh DiRECTIONS}

Given that the focus of the study was on developing the most economically viable scenario for a specific case study, there is the possibility that dissimilar evaluations would have arisen if the focus had been on another case study with different climatic and loading conditions. It is also obvious that the comparison study was limited by the selected MHOAs. In order to resolve these limitations, future work will focus on developing a comprehensive framework for calculating the ranks of a wider range of MHOAs in solving the MG planning problems on a broader level involving the scores obtained by applying them to various MG topologies proposed for different case study sites.

\section{REFERENCES}

[1] T. Ma, H. Yang, L. Lu, and J. Peng, "Technical feasibility study on a standalone hybrid solar-wind system with pumped hydro storage for a remote island in Hong Kong," Renew. Energy, vol. 69, pp. 7-15, 2014.
[2] S. Mohseni and A. Brent, "Smart Grid and Zero-Emissions Energy Systems: The Need for a Multi-Dimensional Investment Planning Perspective," IEEE Smart Grid eNewsl., June 2018.

[3] S. Mohseni, A. Brent, and D. Burmester "The Role of Artificial Intelligence in the Transition from Conventional Power Systems to Modernized Smart Grids," IEEE Smart Grid eNewsl., April 2019.

[4] S. Mohseni and S.M. Moghaddas-Tafreshi, "Development of a multiagent system for optimal sizing of a commercial complex microgrid," arXiv preprint, arXiv:1811.12553, 2018.

[5] B. Zhao, X. Zhang, P. Li, K. Wang, M. Xue, and C. Wang, "Optimal sizing, operating strategy and operational experience of a stand-alone microgrid on Dongfushan Island," Appl. Energy, vol. 113, pp. 16561666, 2014.

[6] N. Ghorbani, A. Kasaeian, A. Toopshekan, L. Bahrami, and A. Maghami, "Optimizing a hybrid wind-PV-battery system using GA-PSO and MOPSO for reducing cost and increasing reliability," Energy, vol. 154, pp. 581-591, 2017.

[7] C. Gamarra and J.M. Guerrero, "Computational optimization techniques applied to microgrids planning: A review," Renew. Sustain. Energy Rev., vol. 48, pp. 413-424, 2015.

[8] D.H. Wolpert and W.G. Macready, "No free lunch theorems for optimization," IEEE Trans. Evol. Comput., vol. 1, pp. 67-82, 1997.

[9] S. Mirjalili, "Moth-flame optimization algorithm: A novel natureinspired heuristic paradigm," Knowledge-Based Syst., vol. 89, pp. 228$249,2015$.

[10] S. Mirjalili, "SCA: A Sine Cosine Algorithm for solving optimization problems," Knowledge-Based Syst., vol. 96, pp. 120-133, 2016.

[11] S. Mirjalili, S.M. Mirjalili, and A. Hatamlou, "Multi-Verse Optimizer: a nature-inspired algorithm for global optimization," Neural Comput. Appl., vol. 27, no. 2, pp. 495-513, 2016.

[12] A. Kaveh and T. Bakhshpoori, "Water Evaporation Optimization: A novel physically inspired optimization algorithm," Comput. Struct., vol. 167, pp. 69-85, 2016.

[13] D. Sharma, P. Gaur, and A.P. Mittal, "Comparative analysis of hybrid GAPSO optimization technique with GA and PSO methods for cost optimization of an off-grid hybrid energy system," Energy Technol. Policy, vol. 1, no. 1, pp. 106-114, 2014.

[14] M.S. Thakur, B. Gupta, V. Kumar, and M. Pandey, "Design and optimization of hybrid renewable energy system (2MWH/D) for sustainable and economical power supply at JEC Jabalpur," Int. J. Curr. Res. Rev., vol. 4, no. 20, pp. 188-197, 2012.

[15] H.W. Teetz, T.M. Harms, and T.W. von Backström, "Assessment of the wind power potential at SANAE IV base, Antarctica: A technical and economic feasibility study," Renew. Energy, vol. 28, pp. 2037-2061, 2003.

[16] M.R. Basir Khan, R. Jidin, and J. Pasupuleti, "Multi-agent based distributed control architecture for microgrid energy management and optimization,” Energy Convers. Manag., vol. 112, pp. 288-307, 2016.

[17] S. Mohseni and S.M. Moghaddas-Tafreshi, "A multi-agent system for optimal sizing of a cooperative self-sustainable multi-carrier microgrid," Sustain. Cities Soc., vol. 38, pp. 452-465, 2018.

[18] N. Yamada and M.N.A. Mohamad, "Efficiency of hydrogen internal combustion engine combined with open steam Rankine cycle recovering water and waste heat," Int. J. Hyd. Energy, vol. 35, pp. 1430-1442, 2010 .

[19] F.A. Farret and M.G. Simoes, Integration of Alternative Sources of Energy. New York: John Wiley and Sons, 2006.

[20] B. Anderson et al., New Zealand GREEN Grid Household Electricity Demand Study 2014-2018. [Data Collection]. Colchester, Essex: UK Data Service. http://dx.doi.org/10.5255/UKDA-SN-853334.

[21] CliFlo: NIWA's National Climate Database on the Web, [Data Collection]. Available: http://cliflo.niwa.co.nz/, Retrieved Dec. 2018.

[22] S. Mohseni and S.M. Moghaddas-Tafreshi, "A multi-agent approach to optimal sizing of a combined heating and power microgrid," arXiv preprint, arXiv:1812.11076, 2018.

[23] S.M. Moghaddas-Tafreshi, S. Mohseni, M.E. Karami, and S. Kelly, "Optimal energy management of a grid-connected multiple energy carrier micro-grid," vol. 152, pp. 796-806, 2019. 